\title{
A prelude report on molecular docking of HER2 protein towards comprehending anti- cancer properties of saponins from Solanum tuberosum
}

Puneet Kumar Singh ${ }^{1}$, Pratyoosh Shukla ${ }^{1,2} *$

${ }^{1}$ Enzyme Technology and Protein Bioinformatics Laboratory, Department of Microbiology, Maharshi Dayanand University, Rohtak-124001, Haryana, INDIA

${ }^{2}$ Birla Institute of Technology (Deemed University), Mesra, Ranchi-835215, Jharkhand, India

*Corresponding author: pratyoosh.shukla@gmail.com

Present Address:

Enzyme Technology and Protein Bioinformatics Laboratory, Department of Microbiology, Maharshi Dayanand University, Rohtak-124001, Haryana, INDIA 


\begin{abstract}
Saponins are extensively known for many biological activities e.g. antimicrobial, antipalatability, anti-cancer and hemolytic. As cancer cells have a more cholesterol-like compound in their membrane structure the saponins bind cholesterol due to their natural affinity to bind cancer cell membrane. This prevents them from entering the body through the intestinal tract where they have the ability to attach themselves to vital organs and grow. This study reports the effective use of lower dose saponins like immunotoxin so that they can inhibit the proliferation of cancerous pancreatic cells. The investigation of pancreatic cancer metabolic pathway it was found that proteins 3H3B produced by genes HER-2 are involved in the enhancement of this type of cancer. Further docking studies showed that there is an effective interaction between saponins and cancer cells. The glide score of the saponin analogue compound with CID 21573770 (Pubchem) was -6.30 followed by score of -6.05 and -5.29 for 5-Florouracil and gemcitabine respectively. The interaction was observed in the GLU and GLN rich region, saponins made H-bonds with GLU-188, GLN-119, VAL-72 and GLN-71. This study indicates an effective way towards leading newer prospects for developing saponin analogue based cancerfighting drugs with improved cancer cell inhibition property without killing normal cells.
\end{abstract}

Keywords: Saponins, molecular docking, anti cancer, pancreatic cancer, Solanum tuberosum 


\section{Introduction}

Pancreatic cancer is a malignant abnormal proliferation of the pancreas. The percentage of developing pancreatic cancer in our lifetime is about 1 in $71(1.41 \%)$ it is same for men and women. Risk factor alters by factor like age, gender, race, smoking, diabetes etc. [1],[2], [3]. The rate of survival of patient suffering from pancreatic cancer is less as compared to other cancers (Government cancer report). There are drugs already available for pancreatic cancer but they all show a lot of side effects. Since during the cancer, patient is already suffering from many problems, so most patient are unable to tolerate the side effect of chemotherapy [4]. In this study we have selected saponins from Solanum tuberosum and its similar structures retrieved from Pubchem which was further compared with available drug i.e. 5-Florouracil and Gemcitabine. Saponins are micro-compound they can be obtained from different sources, each structure obtained from different source is having different structure and they differ in their mechanism too. Around 53 families of plant produce saponins and the number of species is much higher [5]. They are surface active compound which are not observed, they also change the morphology and permeability of cells. Saponins are involved in the normalization of carcinogenic cell proliferation and induce several immune responses rather than a single one. Saponin from soybean is effective for colon cancer [6]. Saponin from different sources differs slightly as it is shown in the saponins from M.sativa and M. truncatula mainly difference found in the more polar region. M.sativa and M. truncatula both possess $(1 \rightarrow 3)$ specific glucosyltransferase but M.sativa possesses $(1 \rightarrow 2)$ specific glucosyltransferase [7]. 


\section{Material and Methods}

Saponins from Solanum tuberosum with its similar structure (20 structures) of around 90\% similarity was retrieved from Pubchem database (http://pubchem.ncbi.nlm.nih.gov/). Pathway was studied in pancreatic cancer from KEGG (http://www.genome.jp/kegg/) (Figure 1) the over expression of HER-2 [8] and point mutations in the K-ras gene was affecting the normal pathway and enhances carcinogenic probability.

Structure of HER-2 (PDB ID 3H3B) was downloaded from Protein Data Bank (http://www.pdb.org/) Docking was performed with GLIDE 8.5. First the preparation of protein as well as of all the legand was done and their energies were minimized. As a reference molecule we have taken 5-Fu and gemcitabine, we compared the result of these drugs with saponins and its similar structure. The docking we did was flexible docking and with default parameters set.

\section{Result and Discussion}

5-Florouracil $(5-\mathrm{Fu})$ is the drug which is being used from the beginning for the treatment of pancreatic cancer but it showed some side effects for example it induces cardiotoxicity and is responsible for dihydropyrimidine dehydrogenase (DPYD) gene germline mutation [9]. In a study conducted 5-Florouracil along with folinic acid and interferon $\alpha$-2a (IFN- $\alpha$ ) has shown no tumor progression in the first two months, it was tested in the $77 \%$ of total patient taken under the trial [4]. A similar kind of test was performed by combination of gemcitabine with 5Florouracil and folinic acid which proved to have a low toxicity profile, this result has been shown by testing the combination of gemcitabine with 6 different forms of 5-Florouracil [10]. 5$\mathrm{Fu} / \mathrm{FA} / \mathrm{IFN}-\alpha$ combination prevented tumor progression initially and it was unclear that whether 
it has positive effect or advantage over 5-Fu alone or with combination with Fu but according to recent studies when the combination of 5-Fu and FA [11]) was given to a patient they resulted effective as compared to gemcitabine. We noted that saponins help to eliminate tumor cells by apoptosis, according to a detailed study of the structure of saponins, sugar side chain influence the tumor cells and with study of all other side chains a results was observed in favour of saponin [12]. We compared the result of 5-Fu and gemcitabine with saponins and their relative structures. It was done on the basis of GLIDE scores. The compound with CID 21573770 (Fig 2a) of pubchem shown the best result i.e. -6.30 followed by the score of 5-Florouracil(-6.05) and gemcitabine (-5.29). CID 21573770 is having molecular weight 868.05882 [g/mol] and its molecular formula is $\mathrm{C}_{45} \mathrm{H}_{73} \mathrm{NO}_{15}$. The interaction between saponins was observed to be in the GLU and GLN rich region, saponins made H-bonds with GLU-188, GLN-119, VAL-72 and GLN-71 making it as an effective interaction.

With this interaction conclusion may refer to have saponins to be more interactive with other two drugs. Since last 12 years gemcitabine is being used over 5-Florouracil because it possesses manageable toxic effects and used as a medical standard for pancreatic cancer [13]. Saponins is showing better results than gemcitabine, though it has been shown in just one respect, further research can prove it to be an effective and non toxicity drug for pancreatic cancer. As stated above a large family of plant produces saponins but saponins biosynthesis in all the family does not depend on the plant order. There are 11 main skeletons identified on which different functional groups and sugar chains are attached to make it individual [14].There are many insilico methods like metabolic pathway study or creation through which effective drug target can be identified and can benefit in the drug discovery process [15]. 


\section{Conclusion}

In the present study we have targeted a single protein HER2 (PDB ID: 3H3B) which is responsible for the enhancement of tumor cell growth. Saponins a secondary metabolite of plants came out to be an efficient chemicals with capability of inhibiting tumor cells. Similarly we can study other targets with study of metabolic pathways and can find most crucial target. Creation of analogs of saponins and screening it on a large scale may provide a promising drug which will bring the quality of life for pancreatic patient. Since many drugs are not permitted through the cell membrane and them posses some other drawbacks, ADME studies can be done with some selective screened drugs. Nevertheless the present docking studies are limited only to certain saponin based analogues but they significantly guide towards new insilico approaches in development of cancer-fighting drugs with improved cancer cell inhibition property

Acknowledgement: The authors gratefully acknowledge the Government of Jharkhand, Department of Agriculture for providing infrastructure development fund to our department. Authors duly acknowledge DBT, Govt. of India for providing BTIS Sub DIC facility for our department. 


\section{References}

1. Pancreatic cancer, American can society, (2011), 1-48.

2. R. J. Stevens, A. W. Roddam, E. A. Spencer, K. L. Pirie, G. K. Reeves, J. Green and A. Beral. Factors associated with incident and fatal pancreatic cancer in a cohort of middle-aged women (2007) British J Cancer 96, 507-509.

3. R. J. Stevens, A. W. Roddam, E. A. Spencer, K. L. Pirie, G. K. Reeves, J. Green and V. Beral. Factors associated with incident and fatal pancreatic cancer in a cohort of middle-aged women (2009) International J Cancer 124, 2400-2405.

4. H. Bernhard, E. J. Arand, G. Bernhard, M. Heike, Klein,J. F. Riemann, K. H. Meyer, W. Dippold and A. Knuth. Treatment of advanced pancreatic cancer with 5-fluorouracil, folinic acid and interferon alpha-2A: results of a phase II trial (1995) British Jounal of Cancer. $71,102-105$

5. S.G. Sparg, M.E. Light, J. V. Staden. Biological activities and distribution of plant saponins (2004) J Ethnopharmacology 94, 219-243.

6. A.V. Rao, M. K. Sung. Saponins as Anticarcinogens (1995) J Nutrition, 717-724S.

7. I. Kapusta, A. Stochmal, A. Perrone, S. Piacente, C. Pizza, and W. Oleszek ,Triterpene Saponins from Barrel Medic (Medicago truncatula) Aerial Parts (2005) J. Agric. Food Chem. 53, 2164-2170.

8. E. Tsiambas, A. Karameris, C. Dervenis, A. C. Lazaris, N. Giannakou, K. Gerontopoulos, E. Patsouris HER2/neu Expression and Gene Alterations in Pancreatic Ductal 
Adenocarcinoma: A Comparative Immunohistochemistry and Chromogenic in Situ Hybridization Study Based on Tissue Microarrays and Computerized Image Analysis (2006) J Pancreas (Online) 7(3), 283-294.

9. A. Shahrokni, M. R. Rajebi, L. Harold, M. W. Saif. Cardiotoxicity of 5-Fluorouracil and Capecitabine in a Pancreatic Cancer Patient with a Novel Mutation in the Dihydropyrimidine Dehydrogenase (2009) J Pancreas 9, 10(2):215-220. Oettle H \& Riess H (2002) Supplement to Cancer 94(4), 912-922.

10. J. P. Neoptolemos, Adjuvant Chemotherapy With Fluorouracil Plus Folinic Acid vs GemcitabineFollowing Pancreatic Cancer Resection (2010) Adjuvant Therapy After Pancreatic Cancer Resection 304(10), 1073-1081.

11. S Man, W Gao, Y. Zhang, L. Huang, C. Liu. Chemical study and medical application of saponins as anti-cancer agents(2010) Fitoterapia 81, 703-714

12. L.Wei. Comparison of current chemotherapy drugs in pancreatic cancer treatment (2010) Journal of Chinese Clinical Medicine 5(6), 351-358.

13. J.P. Vincken, L. Heng, A. Groot, H. Gruppen. Saponins, classification and occurrence in the plant kingdom.(2007) Phytochemistry 68, 275-297

14. L. G. Boros, N. J. Serkova, M. S. Cascante, W. N. P. Lee. Use of metabolic pathway flux information in targeted cancer (2004) Drug Discovery Today: Therapeutic Strategies 1(4). 435-443. 


\section{Figure Captions}

Figure 1. Pathway showing gene enhancing (HER2) the cancer and how it is doing. Source (http://www.genome.jp/kegg/).

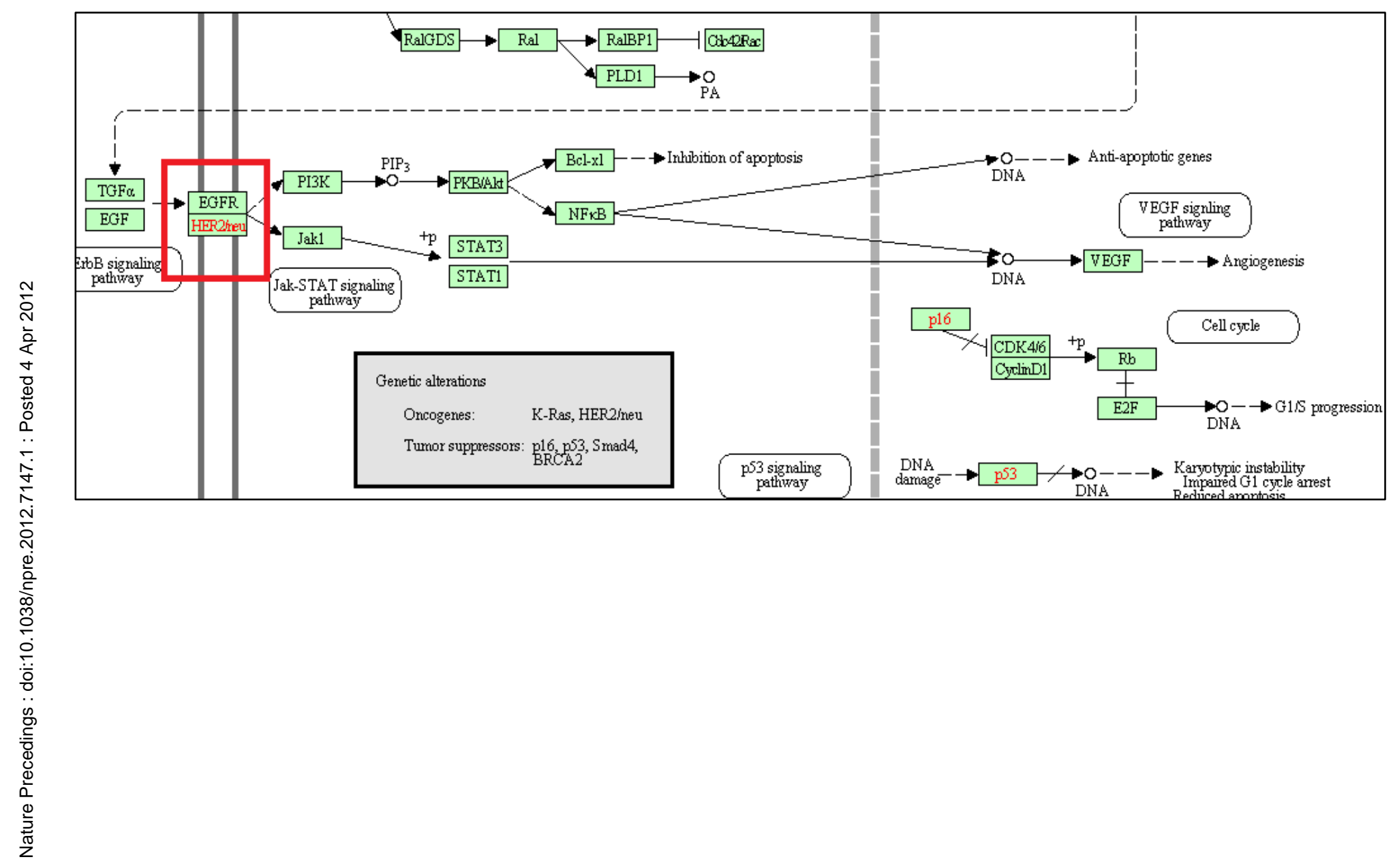


Figure 2. Chemical structures of drugs used for the treatment of Pancreatic cancer (a)CID 21573770, (b) gemcitabine, (c) 5-Florouracil.

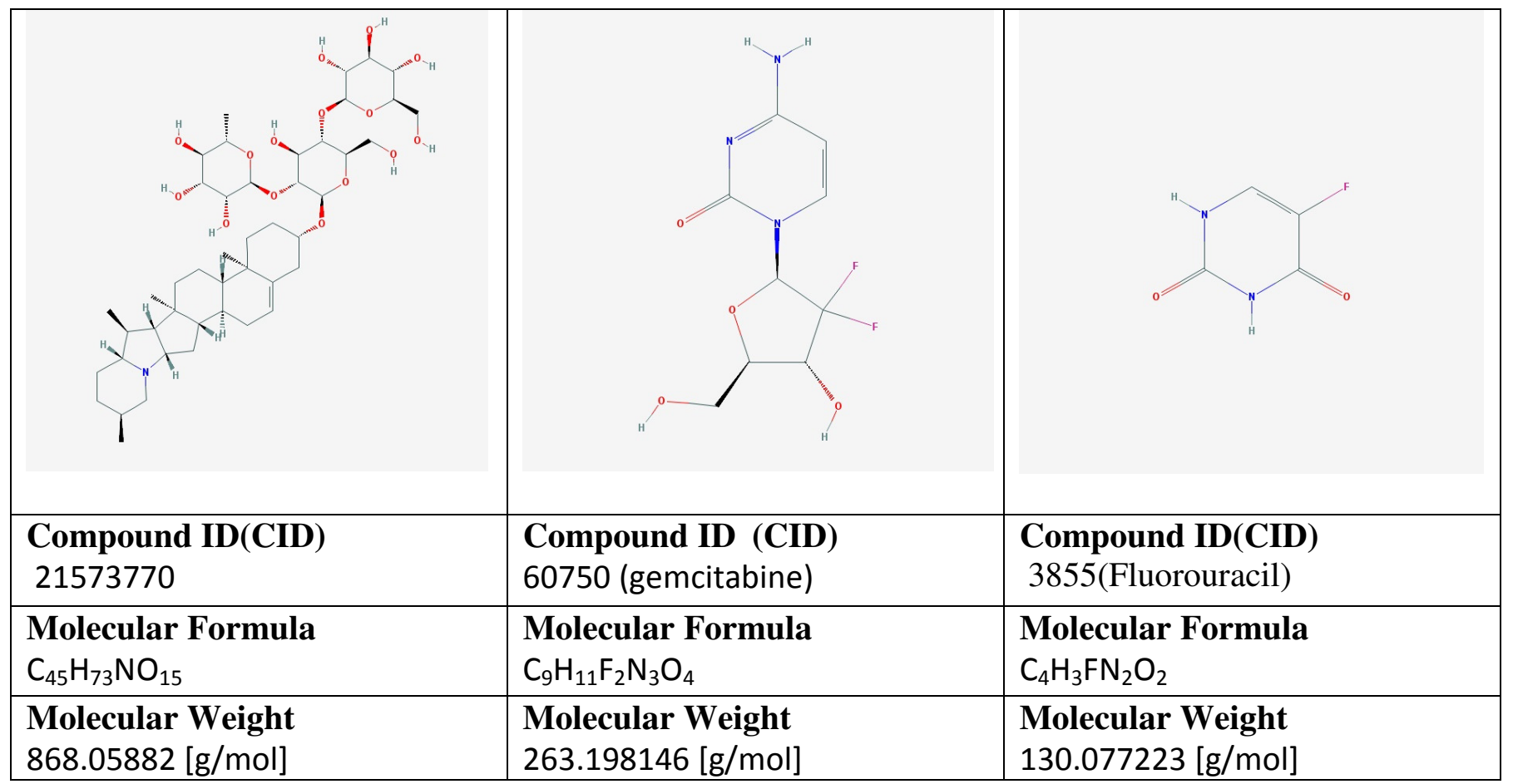


Figure 3. Interaction of HER2 protein with saponins from Solanum tuberosum. H-bonds are formed between GLU-188, GLN-119, VAL-72 and GLN-71 and the protein HER2.

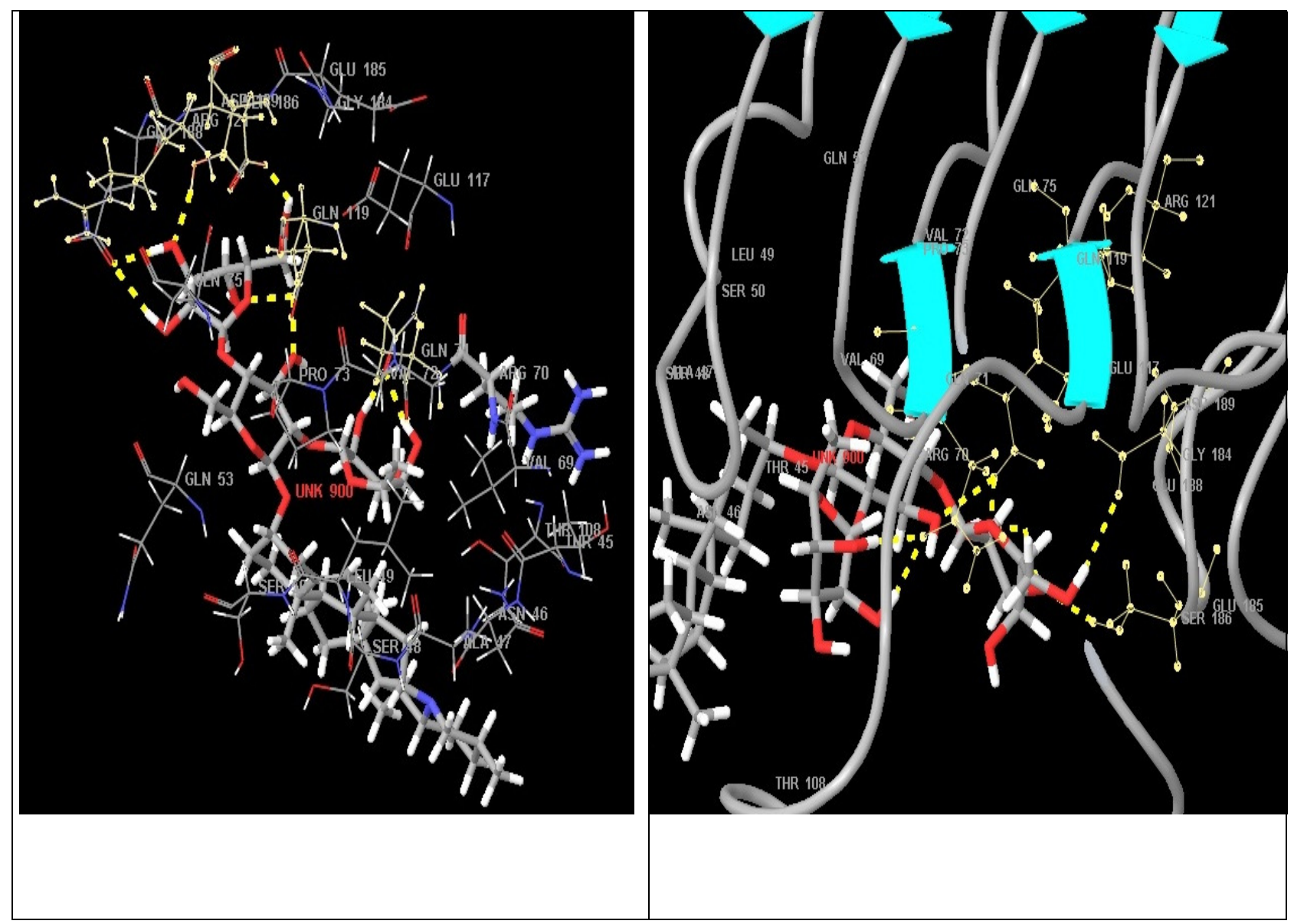

